\title{
A Proposed Fuzzy Stability Model to Improve Multi- Hop Routing Protocol
}

\author{
Hamdy A.M. Sayedahmed \\ Information Technology Department, \\ Central Metallurgical Research \& \\ Development Institute \\ Cairo, \\ Egypt
}

\author{
Hesham A. Hefny \\ Computer and Information Science \\ Department, \\ Institute of Statistical Studies and \\ Research (ISSR) \\ Cairo University, Egypt
}

\author{
Imane M.A. Fahmy \\ Computer and Information Science \\ Department, \\ Institute of Statistical Studies and \\ Research (ISSR) \\ Cairo University, Egypt
}

\begin{abstract}
Today's wide spread use of mobile devices such as: mobile phones, tablets, laptops and many others had driven the wireless Mobile Network growth especially the Mobile Ad hoc Networks commonly referred to as MANETs. Since the routing process is regarded as the core of communication and is associated with the network performance metrics, then its improvement will be revealed in the whole network performance improvement. Due to users' mobility, limited battery power, and limited transmission ranges, the current routing protocols should consider the stability of routes. Hence, the lack of resources of MANETs may result in imprecise routing decisions. In this paper, the proposed fuzzy model is used to handle imprecision of routing decisions by Fuzzy stability model for Dynamic Source Routing (FSDSR). Regarding the number of hops per route, cache size, end-to-end delay and route discovery time, the results showed that FSDSR has outperformed the state of art protocol Dynamic Source Routing protocol (DSR).
\end{abstract}

Keywords-MANET; Fuzzy Model; Routes Stability; OPNET; DSR; FSDSR; MATLAB

\section{INTRODUCTION}

The Mobile Ad-Hoc Network (MANET) is a type of networks that requires no previous infrastructure or centralized control. The application set for MANETs is varying from large/small-scale. Search-and-rescue operations, multi-platform battle deployment, mobile sensors or satellite networks for quick sharing and acquisition of data in inhospitable terrain are examples of these applications as in [10].

One of the challenging issues in MANETs is delivering data packets among mobile nodes in reliably because of MANET's dynamic nature. Multipath routing protocols introduced a solution for that problem by setting a primary route and backup routes. Several Ad-Hoc routing protocols for MANETs were proposed in past years. Most of these routing protocols, such as Destination Sequenced Distance Vector (DSDV), Optimized Link State Routing Protocol (OLSR), Adhoc On-demand Distance Vector Routing (AODV), and Dynamic Source Routing (DSR), were categorized to shortestpath routing protocols which may result in lower throughput and increased packet loss rate. To improve stability of new routes several adaptive protocols were proposed; such as Associativity Based Routing (ABR) and Signal Stability-based Adaptive routing (SSA) as in [4].
The common objective for all routing protocols is to find a stable path between any two communicating nodes with respect to reduction in time complexity and control packet overhead. However, these protocols do not concern stable connections maintenance between the nodes within the network as in [21-24].

This paper is organized as follows: Section II provides an overview of related work. Section III shows Dynamic Source Routing DSR protocol overview. Section IV introduces fuzzy based DSR models. Section V presents the proposed fuzzy stability model. Section VI describes the simulation environment. Section VII discusses the derived results. Section VIII presents the conclusion and future work.

\section{RELATED WORK}

Broadcasting a route request allows a node to discover multiple paths. Reducing the number of requests will reduce the routing overhead that occurs in the route discovery process in AODV, AOMDV, and DSR.An algorithm was proposed in [12] to describe that technique.

Adopting network coding as a mean of improving reliable data delivery decreases control overhead in a large scale networks. The lifetime maximization routing with network coding increases throughput, reduces energy consumption and improves lifetime significantly according to [14], by combining more packets and reducing the number of number of retransmission attempts.

Applying Quality-of-Service (QoS) using Resource Reserve protocol (RSVP) was proposed in [1]. The obtained results showed that DSR and AODV routing protocols had decreased the number of packets dropped.

A priority routing model based on fuzzy closeness approach was introduced in [15]. The derived results had proved that Fuzzy Closeness Based Priority Routing (FCBPR) performs better than DSR in terms of the QOS metrics; packet delivery ratio, throughput, end- to-end delay, jitter routing overhead and normalized routing load.

The proposed protocol in [5] considered two metrics, link and node stability together. Link Expiration Time (LET) and Residual Energy (RE) respectively based on fuzzy logic. The stability level of the entire path can be generated from 
composite metric of these two metrics. The protocol provided minimum control overheads, minimum delay and improved packet delivery ratio by using fuzzy based system.

A fuzzy logic system for caching decisions was introduced in [2], in order to improve routing efficiency. The proposed fuzzy optimal routing algorithm was used to balance the load along multiple paths. It was concluded that paths categorization using fuzzy optimization tended to minimize the disadvantages of both unipath and multipath routing.

\section{DYNAMIC SOURCE ROUTING PROTOCOL}

Dynamic Source Routing - DSR-protocol was classified as a reactive source routing protocol. i.e. it invokes a route discovery mechanism on-demand. Source routing collects the addresses of each node through a link from source to destination. Gathering addresses allow the intermediate nodes to update their route caches as in figure1.

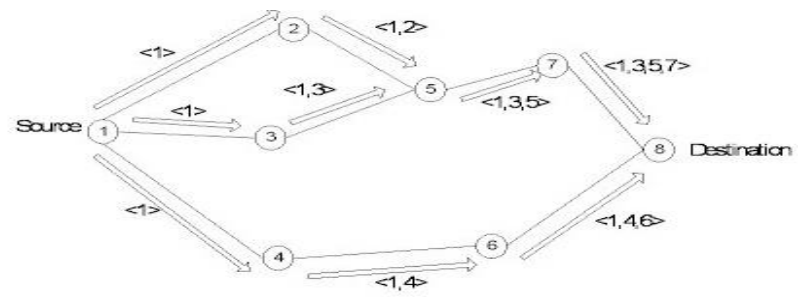

Fig. 1. Sourcing Routing[8]

Also it increases the routing overhead with increasing mobility $[12,23]$. DSR protocol can be composed of two main mechanisms of Route Discovery and Route Maintenance.

\section{A. Route Discovery}

A route request (RREQ) is sent out by source node to discover a route. RREQ builds source route on every path through the network. Each node receives RREQ and has a route to destination it forwards the RREQ to destination node. Also; it drops all RREQs except the first one to the same destination. A node with large number of routes to target node has higher stability than a node that has single route. Destination node receives RREQ and unicast a route reply (RREP) back to sender node via each path.

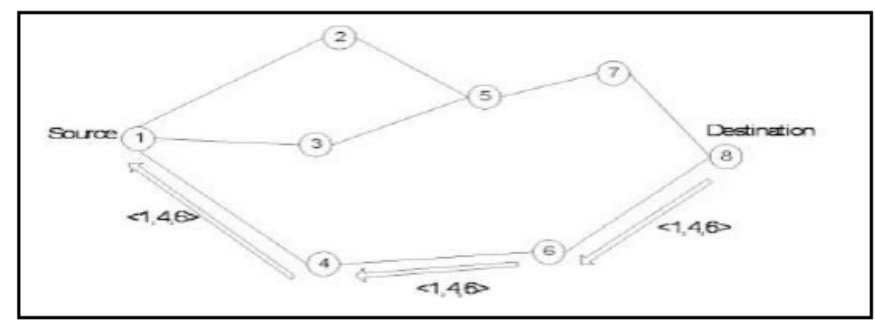

Fig. 2. Single\&Multiple Routes [8]

Figure 2 illustrates that destination node replied to the first RREQ arrived through the link $\langle 1,4,6\rangle$. Node 6 has only single path to node 4 which has single route to the initiator while node 5 has two routes to the initiator. Node 6 may have mobility speed less than node 5 .

\section{B. Route Maintenance}

A route maintenance process starts when a node send a route error (RERR) packet. Originating or forwarding packets are under the responsibility of each node will originate or forward packets to the next-hop. Therefore, a node which discovers a broken link, it will send a RERR to all neighboring nodes. After RERR had been received, each node had sent a packet over that link, it removes this route from its route cache. In figure 3 , node ' $C$ ' discover no route to node ' $D$ '. A node ' $C$ ' sends a packet to node ' $D$ ', if node ' $D$ ' does not send back an acknowledgement packet. It sends out requests until reached the maximum number of times, and then advertises a broken link.

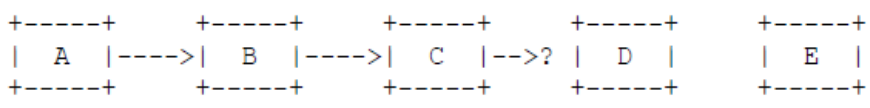

Fig. 3. DSR route broken

\section{FUZZY BASED DSR MODELS}

In [3, 7], Fuzzy logic was introduced by Zaddeh, which is a mathematical technique that deals with imprecise data based on expert knowledge. It is an approach based on "degrees of truth" rather than the conventional computing with a discrete outcome of "true or false" or "1 or 0". A typical fuzzy inference system (FIS) has four basic components as shown in figure 4. Fuzzy logic processes the input parameters to get a decision.

A fuzzy set is characterized by a membership function which has several types such as Gaussian, triangular and trapezoidal. It represents the degree of truth where a set belongs to a linguistic variable. Triangular membership function for $\mathrm{m}$ variable is as equation $1[5,20]$.

$$
\mu(\mathrm{m})=\left\{\begin{array}{cc}
0 & \text { if } m \leq T H 1 \\
\frac{(m-T H 1)}{(T h 2-T H 1)} \text { if } T H 1<m<T H 2 \\
1 & \text { if } m \geq T H 2
\end{array}\right.
$$

Where TH1 is threshold to active system, and $\mathrm{TH} 2$ is threshold to identify level of activeness.

In FIS, a crisp value input converted into a fuzzy singleton with membership function. IF-THEN-rules form a fuzzy rule base. Each input value operates according to corresponding rule. Defuzzifier has the function to map the system output from the fuzzy domain to the crisp domain. Two popular fuzzy models are Mamdani and Sugeno model. The main different of these two models is their own method to generate crisp output. Mamdani FIS generate fuzzy output but Sugeno FIS uses weighted average for the same purpose.

In [16], it was suggested a stable route routing protocol by embedding fuzzy logic system. Based on DSR protocol, when a node wants to transmit data packet to destination node it broadcasts a RREQ packet into a network and when an intermediate node receives the RREQ packet, it calculates input parameters hop count $\left(\mathrm{h}_{\mathrm{i}}\right)$ and stability factor $\left(\mathrm{Sf}_{\mathrm{i}}\right)$ 
currently in path. Then fuzzy logic system evaluates the output parameter fuzzy cost (fc) and determines route is available or not if the route is available, then RREQ is re-broadcasted and the node extracts route record. This process is to be done for each intermediate node until it reaches to the destination. Route reply message generated from destination is sent to source node via the path stored in route record. The rule base of that model is as in table I. Also, a triangular membership function is used in both inputs and output.

The performance analysis showed that fuzzy logic base proposed scheme has better packet delivery ratio and delay than DSR.

TABLE. I. FUZZY WEIGHTED METRICS RULE BASE [16]

Rule1: If hop count is $\mathrm{L}$ and Stability factor is $\mathrm{L}$ then fuzzy cost must be
VH.
Rule2: If hop count is $\mathrm{L}$ and Stability factor is $\mathrm{M}$ then fuzzy cost must be
H.
Rule3: If hop count is $\mathrm{L}$ and Stability factor is $\mathrm{H}$ then fuzzy cost must be
M.
Rule4: If hop count is $\mathrm{M}$ and Stability factor is $\mathrm{L}$ then fuzzy cost must be
H.
Rule5: If hop count is $\mathrm{M}$ and Stability factor is $\mathrm{M}$ then fuzzy cost must be
M.
Rule6: If hop count is $\mathrm{M}$ and Stability factor is $\mathrm{H}$ then fuzzy cost must be
L.
Rule7: If hop count is $\mathrm{H}$ and Stability factor is $\mathrm{L}$ then fuzzy cost must be
M.
Rule8: If hop count is $\mathrm{H}$ and Stability factor is $\mathrm{M}$ then fuzzy cost must be
L.
Rule9: If hop count is $\mathrm{H}$ and Stability factor is $\mathrm{H}$ then fuzzy cost must be
VL.

Fuzzy logic was applied to manage routing policies and enhance routing performance dynamically as in [6]. The parameters signal power, bandwidth, mobility and packet forwarding ratio (PFR) were used as inputs. Gaussian membership function was used for getting the optimal and suitable route based on MANET's inputs parameters, which need to be smooth. The membership function is given by equation 2 .

$$
\operatorname{Gaussian}(\mathrm{x} ; \mathrm{c}, \sigma)=e^{\left(\frac{-1}{2}\right)\left(\frac{x-c}{6}\right)^{2}}
$$
width.

Where c represents MFs center, and $\sigma$ determines the MFs

Also, the output was route optimality. The table II describes the input/output for that model. The obtained performance metrics showed that the overhead had reduced and the routing speed is up.
TABLE. II. INPUT/OUtPUT DETAILS [6]

\begin{tabular}{|c|c|c|c|c|}
\hline \multirow{2}{*}{ Parameters } & \multirow{2}{*}{$\begin{array}{l}\text { Input/Output } \\
\text { Membership } \\
\text { Function }\end{array}$} & \multicolumn{3}{|c|}{ Parameter value } \\
\hline & & $0-0.4$ & $0.2-0.8$ & $0.6-1.0$ \\
\hline Signal power & Input & Low & Medium & High \\
\hline Bandwidth & Input & Poor & Average & Excellent \\
\hline Mobility & Input & Low & Medium & High \\
\hline $\begin{array}{l}\text { Packet } \\
\text { Forwarding Ratio }\end{array}$ & Input & Low & Medium & High \\
\hline Route & Output & $\begin{array}{l}\text { Below } \\
\text { Optimal }\end{array}$ & $\begin{array}{l}\text { Sub } \\
\text { optimal }\end{array}$ & Optimal \\
\hline
\end{tabular}

According to [13], there is still no such model or approach that can provide help in MANET area to compute the behavior of protocols. Therefore, the fuzzy inference system was proposed and modeled for DSR routing protocol by considering some important metrics. The objective of FIS is to reduce the overhead to decide that in which types of network conditions the protocol performs poorly, satisfactory or acceptable. Therefore, tuning the behavior of DSR was implemented by using Mamdani method. A FIS as in figure 4, maps the input variables: Node Density, Pause Time, Node Mobility, Number of Packets transferred, and the Number of Connections, to output variables: Packet delivery Fraction, Normalized Routing Load, and Normalized MAC Load.

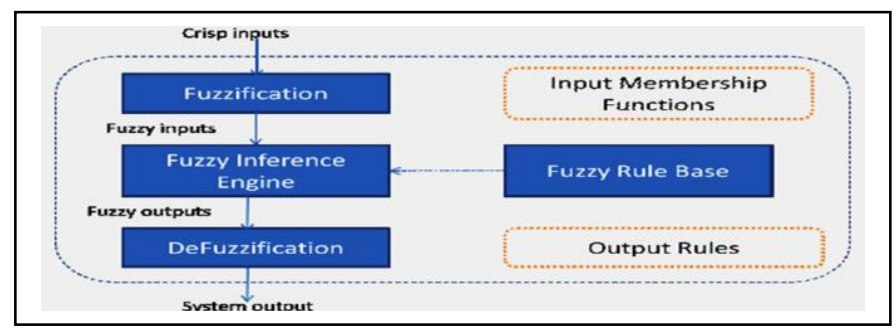

Fig. 4. Low chart of a fis [3]

Also, it was concluded that increasing the number of nodes or changing the speed of mobility, will degrade the DSR 
performance. While number of connection, number of packets, node density, node mobility speed and pause time is increased with proper ratio then DSR performance can be enhanced.

\section{THE PROPOSED FUZZY BASED DSR OPTIMAL PATH STABILITY MODEL}

The proposed model uses fuzzy based decision making technique to determine path stability. As an outcome of fuzzy decision rules, the process of path stability depends on two inputs that represent a node status: total routes in route cache and node speed.

The input variables are total number of routes in route cache (TR) and speed (S). The total routes (TR) is described through 9 linguistic variables $(\mathrm{A}=$ v.low, $\mathrm{B}=$ low, $\mathrm{C}=$ above.low, $\mathrm{D}=$ medium, $\mathrm{E}=$ above.medium, $\mathrm{F}=$ below.high, $\mathrm{G}=$ high, $\mathrm{H}=$ bleow.heavy, $\mathrm{I}=$ heavy), and speed (S) with 7 linguistic variables $(\mathrm{A}=$ resident, $\mathrm{B}=$ move, $\mathrm{C}=$ slow, $\mathrm{D}=$ above.slow, $\mathrm{E}=$ medium, $\mathrm{F}=$ fast, $\mathrm{G}=\mathrm{v}$.fast). The fuzzification membership function for both TR and $S$ is triangular for all linguistic variables as in equation 1 . The universe of discourse for TR and $S$ are $\{0,79\}$, and $\{0,15\}$ respectively. Figures 5 and 6 show the membership function with linguistic variables.

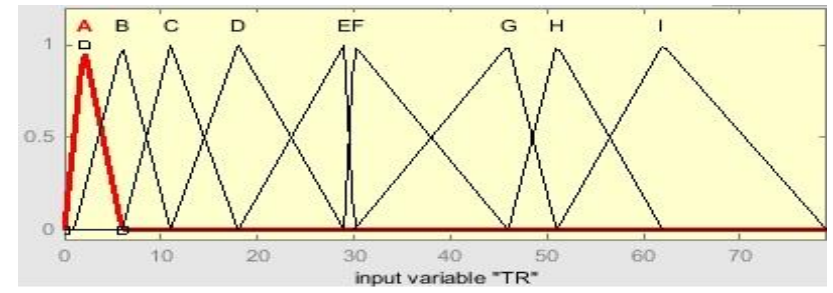

Fig. 5. Input Variable (TR)

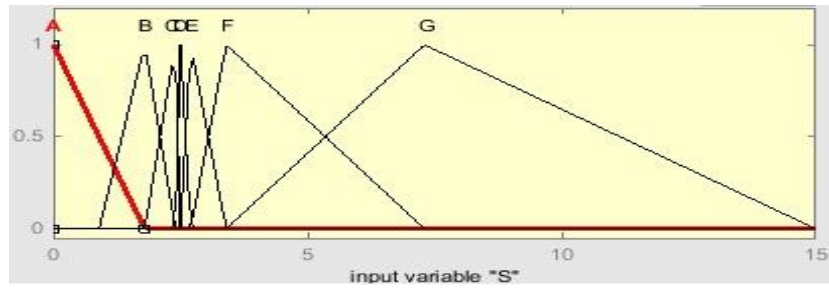

Fig. 6. Input Variable (S)

The FIS type is Mamdani, "And" method is min, "OR" method is max, and the defuzzification is centroid. The "IFTHEN-Rules" are 24 rules as in table IV, which had been obtained from fuzzy clustering algorithm. Fuzzy C-means algorithm (FCM) had been used to cluster collected data set. It had been run with $[6,7,8,9,10,11 \ldots 37,38,39$, and 40] cluster number. Analysis for each run was obtained to get better cluster number and avoid un-discriminated feature(s).

The data set consists of 1497 records that were formed from 16 runs of OPNET Modeler 14.5 using different settings of MANET and default DSR protocol. Data set consists of total number of routes inside each node cache (TR), total number of routes for specified destination inside node cache (NS), the speed of node (S), and the inter-nodes distance between the node and the destination node (D). Also, a default random waypoint had been set for all runs. In this data set, 24 clusters are extracted and inter-nodes distance is not discriminated feature as authors in $[17-19,25]$ guided.

The output variable is the node stability (NS) which represents the number of routes in route cache for specified destination. The node stability (NS) is described through 6 linguistic variables $(\mathrm{A}=$ not.stable, $\mathrm{B}=$ near.stable, $\mathrm{C}=$ below.stable, $\mathrm{D}=$ stable, $\mathrm{E}=$ consistent, $\mathrm{F}=$ v.stable). The defuzzification membership function is triangular for all linguistic variables. Also, the universe of discourse for NS is $\{0,18\}$. Figure 7 shows the membership function with linguistic variables.

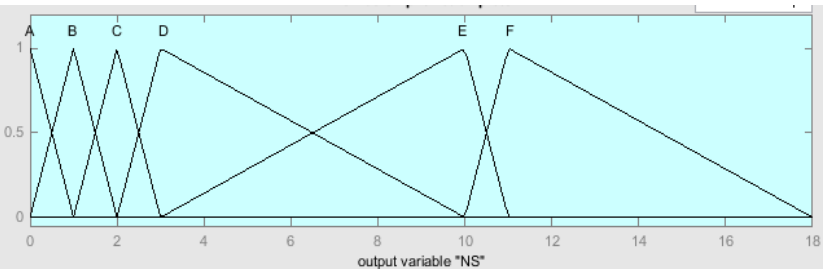

Fig. 7. Output Variable

The proposed fuzzy model $\mathrm{I} / \mathrm{O}$ is as in figure 8 . Also,the model's surface is as figure 9. The objective of fuzzy model is to find the most stable paths. when a source node wants to transmit data packet to destination node it broadcast a RREQ packet into a network and when an intermediate node receives the RREQ packet, it calculates input parameters TR and S currently. Then fuzzy model evaluates the output parameter node stability (NS) and determines node is available or not to participate with that request. If the node is available, then RREQ is re-broadcasted in case a node has no route to destination. This process is to be done for each intermediate node until it reaches to the destination. Route reply packet generated from destination is sent back to source node via the path stored in route record. Each node in each route receives a RREP packet, a fuzzy model calculates the node stability again because it may a node changed its speed or removed routes. If node is available, it forwards the packet to potential destination. Therefore, FSDSR model guarantees that each node along a path has more backup routes which reduce route error RERR. The fuzzy model flowchart is as in figure 15 that describes the process of the fuzzy model.

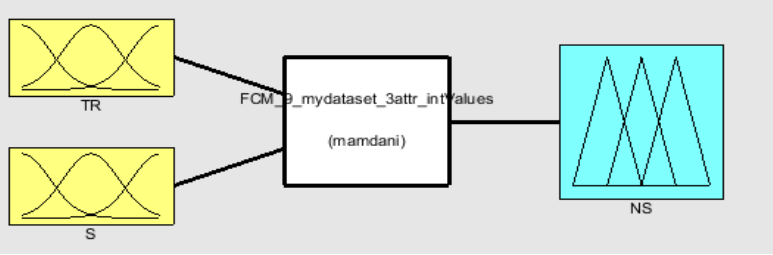

Fig. 8. Fuzzy Model I/O

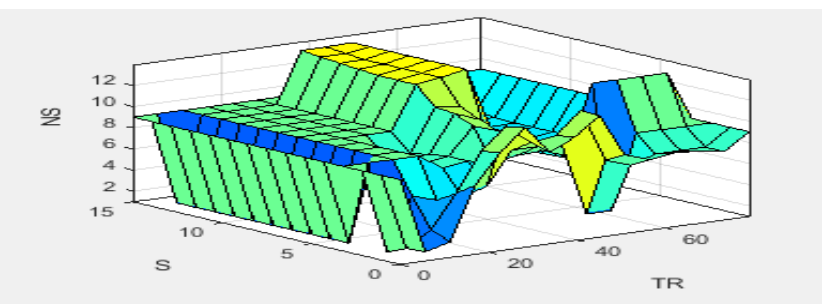

Fig. 9. Fuzzy Model Surface 


\section{SIMULATION ENVIRONMENT}

OPNET Modeler 14.5 and MATLAB R2014b fuzzy toolbox [8] were used to test the proposed fuzzy model. Two scenarios were used to compare standard DSR and Fuzzy Stability Model-DSR (FSMDSR) with table III settings. In addition, the accept condition is at node stability (NS) is ' $\mathrm{E}=$ consistent'.

TABLE. III. SimUlation ATtRIBUtES

\begin{tabular}{|l|l|}
\hline Simulation Parameter & Value \\
\hline Protocol & DSR / FSMDSR \\
\hline Mobility & $\begin{array}{l}\text { Random Waypoint } \\
\text { (Record Trajectory) }\end{array}$ \\
\hline Node Type & "manet_station_adv" \\
\hline No. of Nodes & 15 nodes \\
\hline Area & $1000 * 1000 \mathrm{~m} 2$ \\
\hline Simulation Time & 30 min. \\
\hline
\end{tabular}

As demonstrated by the simulation results from series of experiments for number of hops per route as in figure 10, the number of hops per route represents the number of hops in each route to every destination in the route cache of all nodes. Therefore; a lower number of hops per route lead to the shortest path between two communicating nodes.

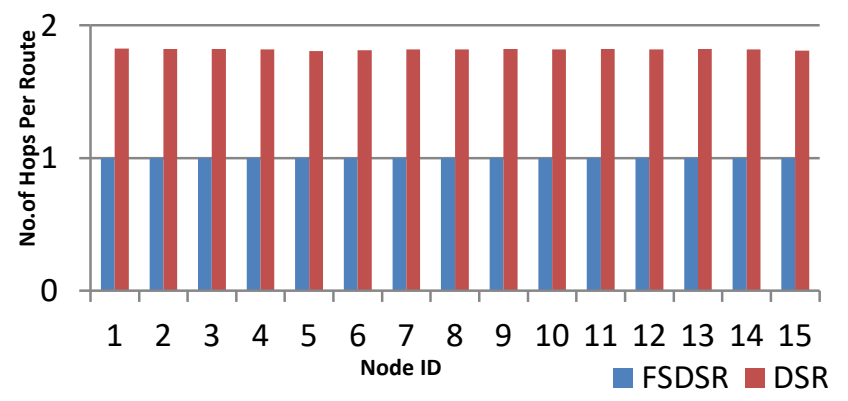

Fig. 10. No. of Hops per Route

Moreover, the route cache size represents the number of routes to different destinations. The node's route cache memorizes all routes which are collected by control packets through source routing. The results as in figure 11 showed the route cache size in the experiment.

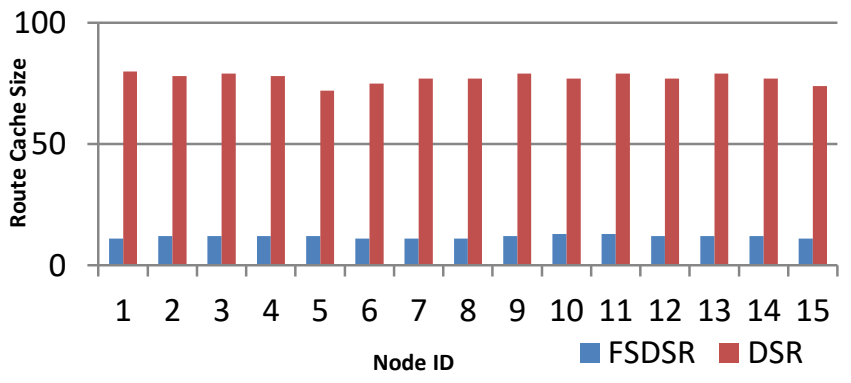

Fig. 11. Route Cache Size

A route discovery time is the time when a route request was sent out until route reply is received. In figure 12 , the route discovery time results.

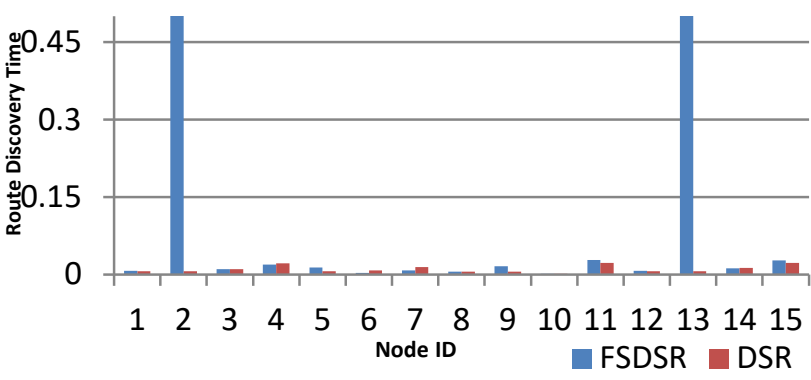

Fig. 12. Route discovery Time

Collected result as is in figure 13 represents the total replies sent from destination which is the total number of route reply packets sent out by all destinations and potentials. The total replies increases with the increase of RREQ.

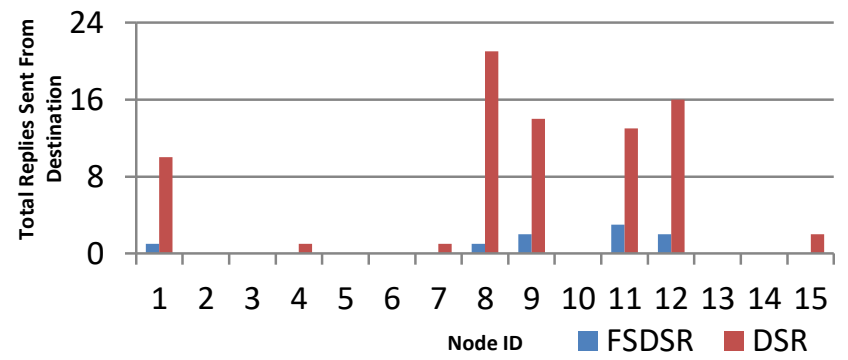

Fig. 13. Total Replies Sent from Destination

Figure 14 represents the wireless LAN delay results through experimental test. Wireless LAN delay is the end to end delay of all the packets received by nodes. Delay increases with increasing the number of control messages exchanging. Wireless LAN delay is expressed as in equation 3 [11].

$$
D_{\text {end to end }}=N\left(D_{\text {trans }}+D_{\text {prop }}+D_{\text {proc }}+D_{\text {queuing }}\right)
$$

Where " $\mathrm{D}_{\text {end to end }}$ is total end to end delay", " $\mathrm{D}_{\text {trans }}$ is transmission delay", " $D_{\text {prop }}$ is propagation delay", " $D_{\text {proc }}$ is processing delay", " $\mathrm{D}_{\text {queuing }}$ is queuing delay", and " $\mathrm{N}$ is the total number of packets"

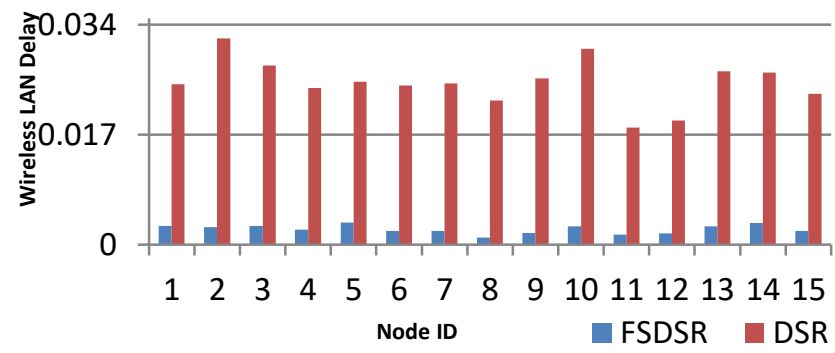

Fig. 14. Wireless LAN Delay

\section{RESULT AND DISCUSSION}

Figure 10 showed that a node which uses FSMDSR will have a lower number of hops per route than when it uses DSR. FSMDSR ( $\mathrm{E}=$ consistent - condition) limits response to a control packets. Therefore, a node that doesn't satisfy the fuzzy model condition, does not reply control packets within that path. Thus, the number of nodes along a path gets decreased. 
On the other hand, DSR has no restrictions on processing all control packets.

In figure 11 , by decreasing number of control packets, the node will decrease the routes in cache as well. Therefore, the node's route cache grows slowly on using FSMDSR. In contrast, overheard un-constrained control packets using DSR results in larger route cache size.

As shown in figure 12, route discovery time is a trade-off when using fuzzy-based MANET routing protocols. Due to each overheared control packet forms a route to be added in node cache. Therefore, node 2 and 13 cannot add all routes which formed from control packets in case of using FSDSR because of FSDSR limitations. Generally, the behavior of FSMDSR on other nodes matches DSR's performance.

In figure 13, the total number of route replies sent from destination in FSMDSR decreased as a result of the lower number of route requests packets and lower cache size. The node that uses DSR could memorize more routes for all its neighboring nodes; therefore, it has a variety of routes for all destinations. However, these routes may be stable or unstable.

Figure 14, by decreasing the number of hops per route in FSDSR, wireless LAN delay is going lower. Since, lower hop number leads to low send/receive control packets between nodes within a certain route. Moreover, it gives evidence that stored routes in route cache (cache size) in FSMDSR always links a source to destination with the lower overhead route.

It can be summarized that FSMDSR outperformed standard DSR for maximum number of hops per route with $45 \%$, for maximum route cache size with $85 \%$, for maximum total replies RREPs sent from destination with $86 \%$, and for maximum wireless LAN delay with $89 \%$. On the other hand DSR outperformed the FSMDSR with 95\% in maximum route discovery time in some cases (node 2 and node 13 ) .

\section{CONCLUSION}

In this paper, a Fuzzy model was proposed to handle the imprecise values of number of routes in route cache and speed of node, to enhance DSR protocol. The derived results proved that the fuzzy model can help to obtain an optimal decision in MANETs.

The proposed fuzzy model (FSMDSR) outperformed the standard DSR in hops per route, total replies sent from destination and wireless LAN delay. Although not all QoS metrics are included in the comparison, it can be concluded that considering the node's route cache and the speed of nodes can improve the performance of Mobile Ad-Hoc network in terms of hops per route total replies sent from destination and wireless LAN delay.

At some node, FSMDSR has a trade-off like in route discovery time (node 2,13). But generally, FSMDSR behaved better in DSR as shown previously.

The future work for this research will consider total cached replies sent route cache size and number of retransmission attempts in order to have a complete view of proposed model and standard DSR. Furthermore, a comparison between FSMDSR and other fuzzy model will be offered.

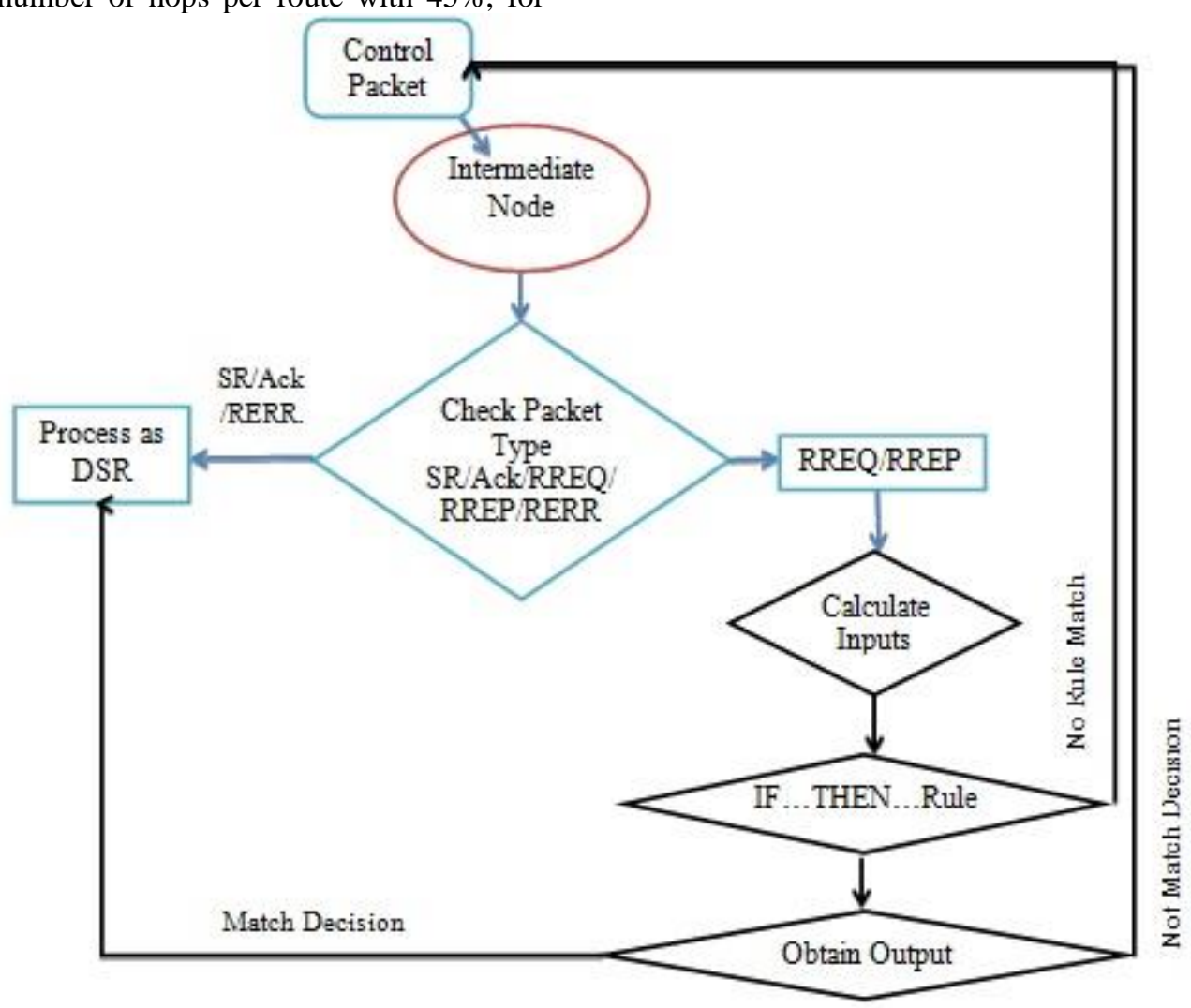

Fig. 15. Fuzzy Model Flow Chart 
TABLE. IV. THE OBtAINED FuZZy IF THEN RULES

\begin{tabular}{|c|c|c|c|c|c|}
\hline IF TR is & v.low & And $\mathbf{S}$ is & resident & Then NS is & not.stable \\
\hline IF $\mathbf{T R}$ is & v.low & And $\mathbf{S}$ is & move & Then NS is & near.stable \\
\hline IF $\mathbf{T R}$ is & low & And $\mathbf{S}$ is & resident & Then NS is & below.stable \\
\hline IF $\mathbf{T R}$ is & low & And $\mathbf{S}$ is & slow & Then NS is & near.stable \\
\hline IF $\mathbf{T R}$ is & above.low & And $S$ is & resident & Then NS is & near.stable \\
\hline IF $\mathbf{T R}$ is & above.low & And $S$ is & above.slow & Then NS is & consistent \\
\hline IF $\mathbf{T R}$ is & medium & And $\mathbf{S}$ is & slow & Then NS is & stable \\
\hline IF $\mathbf{T R}$ is & above.medium & And $\mathbf{S}$ is & resident & Then NS is & v.stable \\
\hline IF $\mathbf{T R}$ is & above.medium & And $S$ is & above.slow & Then NS is & stable \\
\hline
\end{tabular}

REFERENCES

[1] A.A. Arjomand Hashjin, " Implementation a method to provide quailty in manet and survey the effect of that at decreasing Data Dropped of DSR and AODV routing protocols", Scientific Journal of Pure and Applied Sciences, ISSN 2322-2956, January 2015.

[2] A.Gowri, R.Valli, K.Muthuramalingam, "A Review: Optimal Path Selection in Ad Hoc Networks using Fuzzy Logic", International Journal on applications of graph theory in wireless ad hoc networks And Sensor networks, Vol.2, No.4, December 2010

[3] Ai Luang Soo, Chong Eng Tan, Kai MengTay, "A Fuzzy Multiple Metrics Link Assessment For Routing in Mobile Ad Hoc Networks", Global Conference on Power Control and Optimization, Kuching, Malaysia, 2-4, December 2010

[4] Arash Dana, Mohamed Hadi Babaei, “A Fuzzy Based Stable Routing Algorithm for MANET", International Journal of Computer Science, Vol 8, Issues 1, January 2011

[5] Ayushi Singhal , A.K. Daniel, “Fuzzy Logic based Stable On-demand Multipath Routing Protocol for Mobile Ad Hoc Network", Fourth International Conference on Advanced Computing \& communication Technologies, 2014

[6] B.Philo Chaythanya, Dr.M.M. Ramya , “ Fuzzy Logic Based Approach for Dynamic Routing in Manet" , International Journal of Engineering Research \& Technology, ISSN: 2278-0181, Vol. 3 Issue 6, June- 2014

[7] Dr. Ahmad B.Alkhodre, Dr.Mostafa Mahmoud, “ Develop a mobility model for MANETs networks based on fuzzy logic", Journal of Computer Engineering, Vol. 17, Issue 6, Nov-Dec. 2015

[8] https://sites.google.com/site/avicodes/notes/advance-computernetwork/acn-unit6/dsr-the-dynamic-source-routing-protocol-formultihop-wireless-ad-hoc-networks last accessed in Time: 11:00 am, 4 January 2017

[9] https://www.mathworks.com/help/fuzzy/fcm.htmllast accessed in, Time: 11:34 am, 30 October 2016

[10] Jishan Mehedi and Mrinal Kanti Naskar, “A Fuzzy Based Distributed Algorithm for Maintaining Connected Network Topology in Mobile AdHoc Networks Considering Freeway Mobility Model", Journal of Computing and Information Technology , CIT 20, 2012

[11] Mandeep Kaur, Navdeep Kaur, Raju Sharma, "Comparison and Analysis between ReactiveRouting Protocols in MANET using Opnet17.5v ",International Journal of Innovations in Engineering and Technology (IJIET), Volume 6 Issue 1 October 2015

[12] Neha Trivedi, Kumar, Teena Raikwar, "Performance and Evolution of Routing Protocol DSR, AODV and AOMDV in MANET", International Journal of Computer Applications, Volume 109- No. 8, January 2015
[13] Pankaj Sharma, ShrutiKohli, Ashok Kumar Sinha, “ DSR Routing Decisions for Mobile Ad Hoc Networks using Fuzzy Inference System ", The Fourth International Workshop on Computer Networks \& Communications ,October- 2012

[14] T.Manoranjitham, Dr.V.Nagarajan, “ Performance Enhancement Using Network Coding in Dynamic Source Routing”, 3rd International Conference on Recent Trends in Computing 2015 (ICRTC-2015).

[15] V.Tejaswini, K.Chinabusi, "Fuzzy Closeness Based Priority Routing In MANETS", ISSN (PRINT): 2393-8374, (ONLINE): 2394-0697, VOLUME-2, ISSUE-11, 2015

[16] Vivek Sharma, Bashir Alam and M.N. Doja, “ Fuzzy Weighted Metrics Routing in DSR in MANTs", Proceedings Of First International Conference on Information and Communication Technology for Intelligent Systems, Vol.2, 2016

[17] D.Hand, H. Mannila, and P. Smyth, "Principles of Data Mining", MIT Press, 2001.

[18] TeofiloE.Campos, Isabelle Bloch, and Roberto M. "Feature Selection based on fuzzy distances between clusters: First results on simulated data", Springer, 2001

[19] Stephen L.Chiu. "Extracting Fuzzy Rules from Data for Function Approximation and Pattern Classification". Chapter 9 in Fuzzy Information Engineering: A Guided Tour of Applications, ed. D.Dubois, H.Prade, and R.Yager, John Wiley \& Sons, 1997

[20] Bernadette Bouchon-Meunier, MariagraziaDotoli, Bruno Maione. “On The Choice of Membership Functions in a Mamdani-Type Fuzzy Controller",Citeseer, 1996.

[21] BasuDevShivahare, CharuWahi, ShaliniShivahare , " Comparison Of Proactive And Reactive Routing Protocols In Mobile Adhoc Network Using Routing Protocol Property:", International Journal of Emerging Technology and Advanced Engineering, Volume 2, Issue 3, March 2012.

[22] HrituparnaPaul ,PriyankaSarkar , " A Study and Comparison Of OLSR, AODV, and ZRP Routing Protocols In Ad Hoc Networks ", International Journal of Research in Engineering and Technology, Volume: 02 Issue: 08 , Aug-2013.

[23] Mona Gupta and Neeraj Kumar, ' Node-Disjoint On-demand Multipath Routing with Route Utilization in Ad-Hoc Networks ', International Journal of Computer Applications (0975 - 8887) Volume 70- No.9, May 2013.

[24] Arati and Dr.S.S. Tyagi, " Study of MANET: Characteristics, Challenges, Application and Security Attacks', International Journal of Advanced Research in Computer Science and Software Engineering, Vol3- Issue 5, May 2013.

[25] Pang-Ning Tan, Michael Steinbach, Vipin Kumar: Data Mining Cluster Analysis: Basic Concepts and Algorithms, lecture notes, 2006. 